\title{
»a mach nur einen Plan...«: Wege, Umwege und Irrwege zum Frieden im Palästinakonflikt
}

\author{
Margret Johannsen*
}

\begin{abstract}
After the Oslo Process with its gradualistic approach has run aground, the Road Map with its hollow rhetoric has fizzled out and the Geneva Accord with its blueprint for a sustainable peace seems too reasonable to draw support, there is hope that Israel's unilateral disengagement from the Gaza Strip could create renewed momentum in the current Middle Eastern deadlock. However, the Israeli withdrawal from settlements and military installations will be a step towards the twostate solution only if the international community is willing to heavily involve itself, support Palestinian efforts to assure law and order and act against the de facto Israeli annexation of Palestinian territory in the West Bank.
\end{abstract}

Keywords: Israel, Palestine, Road Map, Geneva Accord, Gaza Strip

$\gg P$ ästinensisches Blut ist die rote Linie«, warnte Marwan Barghouti, Chef der Fatah im Westjordanland, aus dem israelischen Gefängnis. Die Warnung war allerdings nicht an Israel gerichtet, sondern an die eigenen Fatah-Leute, die zur Jahresmitte 2004 mit Massendemonstrationen, bewaffneten Überfällen, Entführungen und Brandstiftung Schlagzeilen machten. Über die Zukunft des Gazastreifens ist ein Konflikt entbrannt, der sich an mehreren Fronten abspielt. Israels Ministerpräsident Ariel Scharon hat angekündigt, den Küstenstreifen von der Größe Bremens bis Ende $2005 \mathrm{zu}$ räumen. Aber bis das Militär und die Siedler tatsächlich abziehen, signalisieren die Militäroffensiven der Israelischen Verteidigungsstreitkräfte, dass sie Gaza als Sieger zu verlassen gedenken. Die palästinensischen Milizen wiederum scheinen entschlossen, den Abzug als Niederlage Israels und ihren Anteil daran in die Annalen der Intifada eingehen zu lassen. Sie waren bisher nicht bereit, sich auf eine Waffenruhe zu verständigen. ${ }^{1}$ Der Sicherheitsapparat der Autonomiebehörde (bzw. dessen dezimierter und fragmentierter Rest) sieht sich außerstande, den Kämpfern Einhalt zu gebieten. Die verschiedenen bewaffneten Gruppen führen bereits heute einen Verteilungskampf um künftige Rollen und Ressourcen in den quasi-staatlichen Strukturen. Infolgedessen gelten ihre Angriffe nicht länger allein der israelischen Okkupationsmacht, sondern auch dem palästinensischen Herrschaftsapparat. Loyale Gefolgsleute Arafats werden ebenso attackiert wie Einrichtungen der Autonomiebehörde. Dass allerdings die Regierung in West-Jerusalem diese Situation als Beweis für die Behauptung anführt, es gebe niemanden auf palästinensischer Seite, mit dem man verhandeln könne, ist blanker Zynismus. Denn der palästinensische Sicherheitsapparat ist in erster Linie als Folge der israelischen Militärangriffe auf die Infrastruktur der Autonomiegebiete, insbesondere die Einrichtungen der Ordnungskräfte, zusammengebrochen. Überdies hat die

* Dr. Margret Johannsen, Senior Research Fellow am Zentrum für Europäische Friedens- und Sicherheitsstudien/Institut für Friedensforschung und Sicherheitspolitik an der Universität Hamburg (IFSH).

1 Vgl. Margret Johannsen, Milizen in Palästina zwischen Widerstand und Vgl. Margret Johannsen, Milizen in Palästina zwischen Widerstand und
Opposition, in: Jutta Bakonyi/Jens Siegelberg/Kirsti Stuvoy (Hrsg.), Gewaltordnungen. Ökonomie und Herrschaft jenseits des Staates, Wiesbaden: Sozialwissenschaftlicher Verlag 2004 (i.V.)
Autonomiebehörde in den Augen der Palästinenser nicht nur an Glaubwürdigkeit und Legitimität eingebüßt, weil sie nach Meinung einer großen Mehrheit korrupt ist und der Korruption Beschuldigte unbehelligt bleiben, sondern auch und vor allem, weil ihr Verhandlungskurs gescheitert und der Krieg in die besetzten Gebiete zurückgekehrt ist - daran aber trägt Israel erhebliche Mitverantwortung.

Kann einer der vielen Pläne, die seit 1993 die Schubladen der Diplomaten füllen - u.a. die Oslo-Verträge, die Road Map, das Genfer Abkommen und der Gaza-Abzugsplan den Palästinensern einen eigenen Staat und Frieden bringen? Die meisten von ihnen sind skeptisch. Auch an eine Räumung der jüdischen Siedlungen im Gazastreifen, wie sie Scharons Abzugsplan vorsieht, glaubt nur knapp ein Viertel der Bevölkerung. ${ }^{2}$ Die palästinensische Autonomiebehörde klammert sich an die Road Map des Nahost-Quartetts ${ }^{3}$ und will Scharons Plan als Einstieg verstanden wissen: Den in Aussicht gestellten Abzug aus Gaza sowie die ebenfalls geplante Evakuierung vier isolierter jüdischer Siedlungen im Norden des Westjordanlandes interpretiert sie als Implementierung der von der Road Map geforderten Räumung palästinensischer Gebiete, der weitere Schritte folgen müssten. Dass sie die Road Map hochhält, ist verständlich - was hat sie auch sonst?! Sie hält zwar nur eine Absichtserklärung in den Händen, aber diese ist schließlich auch von den USA unterschrieben, und die Autonomiebehörde weiß so gut wie Israel, dass nur die USA den für eine tragfähige Konfliktregelung erforderlichen Druck ausüben könnten. Dass die USRegierung dies zur Zeit will, kann man allerdings mit Fug und Recht in Frage stellen. Präsident Bush scheint trotz gegenteiliger Beteuerungen selbst nicht mehr an den Plan zu glauben. Jedenfalls hat er das von ihm mitverantwortete Dokument inzwischen desavouiert, als er in einem an die israelische Regierung gerichteten Schreiben vom 14. April 2004 das Rückkehrrecht der palästinensischen Flüchtlinge für obsolet erklärte sowie einen an den Grenzlinien vom

\footnotetext{
2 Palestinian Center for Policy and Survey Research, Poll No. 12, 24-27 June 2004, www.pcpsr.org/survey/polls/2004/p12epdf.pdf.

3 Deutsche Übersetzung unter www.uni-kassel.de/fb10/frieden/regionen/ Nahost/fahrplan-deutsch.html.
} 
4. Juni 1967 orientierten Grenzverlauf zwischen Israel und einem künftigen Staat Palästina ausschloss und damit Entscheidungen traf, die laut Road Map eigentlich zwischen den Konfliktparteien auszuhandeln wären.

\section{Road Map: Unverbindlicher Minimalkonsens}

Die Erwartungen professioneller Optimisten hat die Road Map jedenfalls nicht erfüllt. Sie hatten auf frische Impulse und Durchsetzungswillen des Nahost-Quartetts als neuem Akteur auf der Bühne des ältesten noch virulenten Regionalkonfliktes von internationaler Bedeutung gesetzt. Am 20. Dezember 2002 einigte sich das Quartett auf einen Plan, der den Konfliktparteien innerhalb von drei Jahren den Weg zum Frieden weisen sollte. Das Dokument wurde ihnen am 30. April 2003 offiziell überreicht. 14 Monate später war die Lage unverändert - nur gab es 900 weitere Tote zu beklagen. 2005 wird wohl nicht als das Jahr zu feiern sein, in dem der Dauerkonflikt endete.

Anders als die Oslo-Verträge nennt die Road Map ausdrücklich die Zwei-Staaten-Lösung als Ziel, d.h. einen unabhängigen, demokratischen, lebensfähigen Staat Palästina an der Seite Israels. Das ist ein Vorzug, boten die ergebnisoffenen Vereinbarungen von Oslo doch einst die Möglichkeit, die Augen vor der Logik des Prozesses zu verschließen. Überdies verlangt der Plan den Konfliktparteien Leistungen ohne Vorbedingungen ab. Auch das ist schlüssig, denn Vorbedingungen haben in der Vergangenheit allzu oft den politischen Prozess blockiert. Das Dokument repräsentiert den kleinsten gemeinsamen Nenner zwischen den Konfliktparteien wie auch zwischen den Mitgliedern des Quartetts. Der Konsens war aber nur um den Preis gravierender Mängel erreichbar, was Verbindlichkeit des Kalenders, Klarheit der Zielvorgaben und Durchsetzungswillen des Quartetts angeht. Vermutlich wird den Plan des Nahost-Quartetts darum das Schicksal seiner Vorgänger ereilen und die Road Map wird nur als vergeblicher Anlauf der Diplomatie, nicht aber als Wegweiser zum Frieden in die Geschichtsbücher eingehen.

Die Gewalt ging jedenfalls weiter, der Blutzoll betrug durchschnittlich zwei bis drei Tote am Tag - ein Zermürbungskrieg ist das nicht, denn diese Anzahl können beide Seiten verkraften. Die palästinensische Autonomiebehörde, die Widerstand fürchtete, wenn sie versuchen würde, die militanten Gruppen auf die vagen Versprechungen der Road Map hin zu entwaffnen, bemühte sich stattdessen, mit ihnen eine Waffenruhe zu vereinbaren, in die sie Israel einzubeziehen hoffte. Die israelische Regierung beharrte indes von Anfang an darauf, dass die Milizen zerschlagen würden, bevor sie ihrerseits bereit wäre, ihre Verpflichtungen zu erfüllen. Solange sah sie sich berechtigt, die militärischen Operationen, die Blockade der Städte und den Siedlungsbau fortzusetzen.

Vor allem trieb Israel die Errichtung der Sperranlage voran, die Sicherheit vor palästinensischem Terror verspricht. In den Augen der Palästinenser stellt die »Apartheid-Mauer« hingegen ein Instrument zu weiterem Landraub dar, da sie östlich der Waffenstillstandslinie von 1948 verläuft und teilweise weit auf palästinensisches Gebiet vordringt, um jüdische Siedlungen einzuschließen. Ob Israel das monströse Bauwerk nach dem Spruch des Internationalen Gerichtshofs in Den Haag, der es am 9. Juli 2004 für völkerrechtswidrig erklärt hat, wie gefordert tatsächlich abreißt und auf der Grünen Linie wiedererrichtet, ist mehr als zweifelhaft. Das Rechtsgutachten ist jedenfalls ebenso wenig bindend wie die ihm folgende Resolution der UNO-Vollversammlung vom 20. Juli 2004, und der Sicherheitsrat wird es nicht übernehmen - dafür dürfte gegebenenfalls ein amerikanisches Veto sorgen. Die Legitimität des »Anti-Terror-Zauns « steht für den israelischen Premier wie für die Mehrheit seiner Landsleute außer Zweifel, auch wenn nicht jeder die Entgleisung des Regierungschefs billigen dürfte, der zwei Tage nach dem Richterspruch den ersten Terroranschlag seit April 2003 in Tel Aviv zum Anlass nahm, um die Richter zu dessen Schirmherren zu erklären. Mehr als ein paar Korrekturen hier und da, bereits Ende Juni 2004 vom Obersten Gericht Israels verfügt, wird die Regierung mit Sicherheit nicht vornehmen, wenn man sie gewähren lässt.

Eine Sanktionsinstanz, die Verstöße der Parteien gegen ihnen auferlegte Pflichten - Unterlassungen oder gar Sabotageversuche - ahnden könnte, sieht die Road Map nicht vor. Sie fordert im Gegenteil Störmanöver - sei es von Regierungen, sei es von substaatlichen Gruppierungen - geradezu heraus. ${ }^{4}$ Denn die Feststellung des Quartetts, dass die Parteien ihre Verpflichtungen nicht erfüllen, bedeutet Stillstand auf dem Weg zu einer abschließenden Regelung und belohnt infolgedessen die Obstruktionskräfte. Das NahostQuartett sollte darum einen verbindlichen Zeitrahmen für die Konfliktlösung setzen. Dafür muss allerdings über deren Elemente Klarheit bestehen. Über die Kernfragen eines völkerrechtlich gültigen Vertrages, der diese abschließend regelt, sollten umgehend Verhandlungen beginnen. Als Bezugsrahmen kann das Genfer Abkommen ${ }^{5}$ dienen.

\section{Genfer Abkommen: Blaupause für einen tragfähigen Friedensschluss}

Politiker und Experten beider Seiten haben diese »Blaupause« für eine vertragliche Zwei-Staaten-Lösung ${ }^{6}$ in der Absicht entwickelt, eine praktikable Friedensvision zu präsentieren, dafür internationale Unterstützung zu mobilisieren und so den Menschen in der Region den Glauben an eine Alternative zu Terror und Militarismus zurückzugeben. Die Initiatoren präsentierten ihr Werk am 1. Dezember 2003 in Genf. Wie auch die Gründer von People's Voice, die in beiden Gesellschaften Unterschriften für die Zwei-Staaten-Lösung sammeln ${ }^{7}$, setzen sie auf einen »von unten« initiierten Politikwechsel. Eine gesellschaftliche Debatte über die vielbeschworenen »schmerzhaften « Kompromisse zu entfachen,

4 Vgl. Margret Johannsen, Friedenspläne und ihre Feinde im israelischpalästinensischen Konflikt, in: Christoph Weller et al. (Hrsg.), Friedensgutachten 2004, Münster: Lit 2004, S. 79-87.

5 Deutsche Fassung unter www.genfer-initiative.de>.

6 Vgl. Muriel Asseburg, Von Aqaba nach Genf - Herausforderungen für europäische Politik, in: Vierteljahresschrift für Sicherheit und Frieden $(\mathrm{S}+\mathrm{F})$, 3-4/2003, S. 121-127.

7 Statement of Principles, signed by Ami Ayalon \& Sari Nusseibeh on July 27, 2002, www.mifkad.org.il/eng/PrinciplesAgreement.asp. 
den Obstruktionskräften in beiden Gesellschaften eine aktive Bewegung für pragmatische Regelungen entgegenzusetzen und den politischen Führungen das Mandat zu erteilen, tragfähige Lösungen auszuhandeln - das ist der Weg, der den Initiatoren des Genfer Abkommens vorschwebt. Es setzt auf internationales Engagement: bei der Streitschlichtung, der Aufnahme von Flüchtlingskontingenten, der Übernahme von Kosten für deren Ansiedlung und Entschädigung, der Überwachung des israelischen Truppenrückzugs, dem militärischen Schutz des palästinensischen Staates etc. Mit internationalem Engagement steht und fällt die Lösung des Konflikts. Nur wenn die unmittelbar betroffenen Menschen überzeugt werden, dass die Staatengemeinschaft ihren Part auch spielen wird, werden sie den Glauben an die Möglichkeit einer ausgehandelten und fairen Konfliktlösung wiedergewinnen. Dafür allerdings brauchen sie unmissverständliche Signale. Wer die zivilgesellschaftliche Initiative in ihrem Bemühen unterstützt, einen friedenspolitischen Richtungswechsel in Gang zu setzen, gibt zu verstehen, dass er sich seiner Verantwortung stellen wird, wenn ein Vertragswerk vor Ort Realität werden soll. Angesichts der dramatischen Verschlechterung der Lage sind auch die Deutschen gefordert - nicht nur eingedenk ihrer historischen Mitverantwortung für den Palästina-Konflikt, sondern auch und vor allem aus eigenem Interesse an Frieden in ihrer Nachbarregion.

\section{Abzug aus Gaza und Mauerbau im Westjordanland}

Was Scharon mit seinem Abzugsplan vorgelegt hat, ist allerdings mitnichten ein Vertragswerk und zielt erklärtermaßen nicht auf einen verhandelten und für beide Seiten akzeptablen Frieden ab. Dennoch ist nicht auszuschließen, dass der Plan Bewegung in die festgefahrene Lage bringt. Der Abzug wird allerdings nur einen Schritt hin zu einer tragfähigen Konfliktlösung darstellen, wenn durchsetzungsfähige und -willige internationale Akteure ihn begleiten und überdies verhindern, dass im Gegenzug - wie von Scharon beabsichtigt - die israelische Landnahme im Westjordanland zementiert wird. ${ }^{8}$ Wenn im Gazastreifen ein Gemeinwesen mit legitimen Institutionen entsteht, das im Interesse der Bürger für Recht und Ordnung sorgen und Angriffe gegen Israel unterbinden kann, besteht vielleicht die Chance, die Zirkel von Gewalt und Gegengewalt zu durchbrechen und die von der Road Map anvisierte Zweistaatlichkeit wieder auf die Tagesordnung zu setzen. Die Staatengemeinschaft kann diesen Prozess fördern, indem sie der palästinensischen Autonomiebehörde hilft, sich als Führung im Gazastreifen mit breitem Rückhalt in der Bevölkerung zu etablieren. Eine internationale Präsenz würde signalisieren, dass das Quartett über seine Beobachterrolle hinaus bereit ist, substanziell Verantwortung zu übernehmen. Es gibt bei den Palästinensern klare Mehrheiten für eine bewaffnete internationale Truppe, um die ägyptisch-palästinensische Grenze und den internationalen Grenzübergang in Rafah zu sichern und

8 Vgl. Muriel Asseburg, Abzug aus Gaza?, in: Blätter für deutsche und internationale Politik, 7/2004, S. 781-784. ebenso klare Mehrheiten für eine internationale Präsenz, die helfen soll, die Institutionen der Selbstverwaltung, die Wirtschaft und die Infrastruktur wiederaufzubauen. Mit einem technischen Wiederaufbau ist es aber nicht getan. Um die Selbstverwaltung mit demokratischer Legitimität zu versehen, sind nicht Statthalter, sondern Wahlen erforderlich. Sie werden von Organisationen der Zivilgesellschaft, z.B. von der im Juni 2002 gegründeten Palästinensischen Nationalen Initiative als Voraussetzung für demokratisch legitimierte Verhandlungen mit Israel angemahnt, ${ }^{9}$ setzen allerdings ein Mindestmaß an Freizügigkeit und Sicherheit voraus. Wenn Kandidaten Grund zur Annahme haben, bei Wahlveranstaltungen liquidiert zu werden oder wenn Wähler fürchten müssen, dass Wahllokale bombardiert werden, lassen sich keine Wahlen abhalten, aus denen eine neue politische Führung Legitimität beziehen kann.

In die Wiederherstellung einer funktionsfähigen Selbstverwaltung sind kooperationsbereite Strukturen vor Ort einzubeziehen. Wenn die national-religiöse Islamische Widerstandsbewegung Hamas - die nicht nur aufgrund ihres bewaffneten Kampfes, sondern auch wegen ihrer sozialen Dienstleistungen populär ist - ausgeschlossen wird, dürften die Hilfsmaßnahmen ihren Zweck verfehlen, das politische System zu stabilisieren. Eine überwältigende Mehrheit der Palästinenser wünscht, dass die Hamas nach Abzug der Israelis an der Administration des Gazastreifens gleichberechtigt beteiligt wird und bei den überfälligen Kommunal-, Parlaments- und Präsidentschaftswahlen kandidiert. Die Bewohner der besetzten Gebiete sind in ihrer großen Mehrheit Anhänger der Zwei-Staaten-Lösung, scheinen sich aber trotzdem nicht von der Hamas-Charta, in der die Organisation sich die Befreiung Palästinas vom Jordan bis zum Mittelmeer auf ihre Fahnen geschrieben hat, abschrecken zu lassen. Vermutlich setzen sie auf den Pragmatismus der Islamischen Widerstandsbewegung. Deren spirituelles Oberhaupt Scheich Achmed Yassin hatte unlängst eingestanden, dass die »Befreiung ganz Palästinas « wohl nur schrittweise möglich sei. Diese Äußerung lag in einer Linie mit wiederholten Erklärungen hoher Funktionsträger seit 1988, dass mit einem Rückzug Israels auf die Grenzen von 1967 der bewaffnete Kampf beendet sei. Seit dem Tode Yassins sind die gemäßigten Stimmen allerdings vorerst verstummt. ${ }^{10} \mathrm{Ob}$ seine "gezielte Tötung ${ }{ }^{11}$ - wie die von Israels Oberstem Gericht bisher nicht beanstandeten Liquidierungen im Regierungsjargon heißen - den Konflikt einer politischen Lösung näher bringt, ist mehr als zweifelhaft. Yassin war kraft seiner Autorität als Gründer und geistliches Oberhaupt der Hamas eher als jeder andere in der Lage, die Organisation gegen den Widerstand von Hardlinern in eine konstruktive Kraft in der Selbstverwaltung oder zumindest eine loyale Opposition zu transformieren. Die Liquidierung von Mitgliedern der Führungsriege indes birgt die Gefahr einer Zersplitterung

9 Mustafa Barghouti, Palestinian Elections: - the only hope for legitimate peace talks, 13. Dezember 2003, www.palestinemonitor.org/mustafa/palestinian_elections.htm.

10 Helga Baumgarten, Hamas: Soziale Integration und bewaffneter Widerstand, in: INAMO, 38/2004, S.46-50.

11 Vgl. Margret Johannsen, Das Exempel Jassin oder Wie Ariel Scharon Recht behält, in: Blätter für deutsche und internationale Politik, 5/2004, S. 605613. 
der Bewegung. Wenn die Kontrolle über einzelne Zellen und Aktivisten verloren geht, wird künftig jede Waffenruhe noch schwieriger durchzusetzen sein. Ein Israel »unter Feuer« aber kann sich der schützenden Hand seines Schirmherrn jenseits des Atlantiks sicher sein, was immer es auch zu seiner vermeintlichen Sicherheit in den besetzten Gebieten unternimmt. Vielleicht ist das ja der tiefere Sinn von Scharons Liquidierungspolitik.

\title{
»Und weil der Mensch ein Mensch ist...«
}

\author{
Interkulturelle Ethik, religiöse Identität und Konflikt
}

\author{
Heinrich Schäfer*
}

\begin{abstract}
Many contemporary conflicts are characterized by strong religious and cultural factors. This is where ethical principles like the discursive »moral principle« of Karl - Otto Apel enter in difficulties. Intercultural ethics has to deal with the network of cultural and religious convictions of the actors implied in conflicts. The present article shows a method of how to link to such a network of a fundamentalist movement and to introduce slight but effective changes. But the concentration on thick cultural convictions - as communitarianists do - does not mean to leave basic humanitarian principles aside. The reflection on the preconditions of liberal ethical principles in bodily human life allows for an interculturally valid reference to a principle of universal humanity.
\end{abstract}

Keywords: Ethics, intercultural relations, identity networks, liberalism, communitarianism

$\gg \mathrm{U}$ nd weil der Mensch ein Mensch ist, drum braucht er was zu essen, bitte sehr...« Pragmatisch, schnörkellos und ohne Umschweife - wie es Brechts Art ist - begründet hier das »Einheitsfront-Lied « die Forderung nach der Befriedigung eines Grundbedürfnisses mit einem schlichten Hinweis. Es reicht, dass der Mensch ein Mensch ist, damit diese Forderung gilt - universal selbstverständlich.

Dass weder Einheitsfronten noch Vereinheitlichung unter dem Marktmodell in Sachen Grundbedürfnisse aller Menschen komplett überzeugen konnten, ist für die Wirtschaftsethik der Globalisierung ein aktuelles Thema. Der Verweis auf das Menschsein des Menschen hat allerdings noch eine andere interessante Seite. Sie wird sichtbar, wenn es darum geht, Ethik im Kontext interkultureller Differenzen und Konflikte zu entwerfen. Auf diesen Aspekt möchte ich mich in diesem Vortrag konzentrieren.

Unterschiede und Konflikte zwischen Kulturen gewinnen eine immer stärkere sicherheitspolitische Bedeutung. Die ethnisch-religiösen »neuen Kriege - die bewaffneten Stiefbrüder der Globalisierung - stellen neue Anforderungen. Der Bundesverteidigungsminister hat ja erst in seiner Regierungserklärung vom 11. März darauf hingewiesen, dass in den verschiedensten »Einsatzformen - von der Patrouille am Horn von Afrika über zivilmilitärische Projekte bis zur Beobachtung in Georgien -,, Konfliktverhütung, Krisenbewältigung und friedensstabilisierende Maßnahmen immer

\footnotetext{
* Prof. Dr. theol. habil. Dr. phil. (rer. soc.) Universidad Nacional, Costa Rica, und Universität Hannover (Lehrbeauftragter). Der Vortrag wurde am 1. Mai 2004 an der Fakultät für Pädagogik der Helmut SchmidtUniversität, Hamburg, gehalten. Der Vortragsstil wurde beibehalten.
}

wichtiger werden. Da ist interkulturelle Kompetenz gefragt. Was heißt das für philosophische und theologische Ethik? Das Problem ist schnell eingegrenzt: Wie immer wieder gesagt wird, ist es nicht leicht, ethische Standards oder Prinzipien über die Grenzen von Kulturen hinweg zu vermitteln trotz (oder gerade wegen?) der erheblichen Raum-ZeitKompression in der jüngsten Globalisierungsphase. Ich nenne nur die Debatte um westliche versus östliche Menschenrechte.

Nun hat Karl-Otto Apel - als einer der Begründer der Diskursethik - bekanntlich ein großes Interesse an universalen Moralprinzipien. Er bewertet sie deutlich höher als partikulare, kontextualistische Ethiken des guten Lebens. Aber er sieht zugleich drei schwere, ungelöste Probleme, wenn es darum gehen soll, dass Menschen anderer Kulturen sich einlassen auf Vernunftprinzipien wie etwa den kategorischen Imperativ Kants oder das diskursethische Moralprinzip. Diese Probleme sind:

- Der Mangel politischen Willens, etwa wenn Fundamentalisten ihre Ausschlusslogiken vertreten;

- Probleme hermeneutischer Verständigung; sowie

- soziale Gegensätze und Konflikte, die Verständigungsbemühungen überlagern.

Ein wahrer Dschungel von Schwierigkeiten! Sie behindern, so Apel, die Anwendung eines universalen Vernunftprinzips. Das stimmt.

Aber gerade interkulturelle Konfliktsituationen können uns ja auch auf den Gedanken bringen, die Problemstellung umzukehren. Was man gemeinhin als »Anwendungsprobleme« von Ethik kennt, wäre dann - mit den Worten von Julian 